\title{
Vascularidad Arterial Extraglandular de la Próstata del Perro
}

\author{
Extra Glandular Arterial Vascularity of the Dog Prostate
}

\author{
Ricardo Olivares* \& Mireya Aguayo*
}

OLIVARES, R. \& AGUAYO, M. Vascularidad arterial extraglandular de la próstata del perro. Int. J. Morphol., 32(4):1277-1281, 2014.

RESUMEN: La próstata es la glándula accesoria sexual más importante del perro, ubicada en el canal pélvico, retroperitoneal y caudal a la vejiga, envolviendo completamente la uretra pélvica en su salida del cuello vesical. Su función es producir líquido seminal que provea de un medio ambiente óptimo para la sobrevida y motilidad espermática. Pudiendo verse afectada por una serie de procesos patológicos tales como: Hiperplasia prostática benigna y neoplasias. El objetivo del presente trabajo, fue describir la distribución de los vasos sanguíneos arteriales extraglandulares de la próstata canina, como un factor de importancia y utilidad, para la comprensión del control fisiológico y de ciertos procesos patológicos de dicha glándula, para su posterior abordaje médico-quirúrgico. Se utilizaron 5 machos caninos mestizos adultos, entre 3 y 5 años de edad, y de 15 a $20 \mathrm{~kg}$ de peso, sin patologías prostáticas diagnosticadas, eutanasiados en centros veterinarios particulares y públicos. Los animales fueron perfundidos por la Arteria carótida común con solución fijadoraconservadora, para luego de $72 \mathrm{~h}$, inyectar por la misma vía, látex coloreado rojo, manteniendo posteriormente los cuerpos en refrigeración hasta la realización de la disección regional. Tomando como referencia lo indicado por algunos autores, se pudo determinar que independiente de las ramificaciones de la Arteria prostática, ésta irriga a la glándula, siempre por medio de tres ramas vasculares.

PALABRAS CLAVE: Próstata; Irrigación; Perro.

\section{INTRODUCCIÒN}

La próstata, es la glándula accesoria sexual más importante del macho canino, está ubicada en el canal pélvico, retroperitoneal y caudal a la vejiga, envolviendo completamente la uretra pélvica en su salida del cuello vesical (Gobello \& Corrada, 2002; Smith, 2008; Thibaut et al., 2009). En la pubertad, la glándula se localiza en la cavidad pélvica, pero en un alto porcentaje de perros de más de cinco años de edad, se producen variaciones de tamaño ubicándose en el abdomen (Gobello \& Corrada; Dimitrov et al., 2010).

Anatómicamente, la próstata es una estructura glándulomuscular semioval (Dimitrov et al.), que presenta dos lobos bien diferenciados entre sí, debido a la presencia de un rafe medio de situación dorsal (Gobello \& Corrada; Smith) y comienza a desarrollarse hacia la sexta semana de gestación (Kustritz, 1997). La Nómina Anatómica Veterinaria (NAV) (International Committee on Veterinary Gross Anatomical Nomenclature, 2005), describe un cuerpo de la próstata, el que está conformado por dos lobos, un istmo y dependiendo de la especie, una parte diseminada.
Los vasos sanguíneos de la próstata se encuentran bilateralmente en la superficie dorso lateral de la glándula (Freitag et al., 2007). Las primeras descripciones de la vascularización de la próstata canina datan de Gordon en 1960 y Hodson en 1968, citados por Stefanov (2004).

El aporte arterial de la próstata deriva de la Arteria (A.) ilíaca interna, que se continúa en la A. pudenda interna, la cual es un vaso más pequeño y ventral que corre en dirección caudal sobre el tendón terminal del Músculo (M.) psoas menor. A nivel de la Articulación (Art.) sacro ilíaca, esta última da origen a la A. prostática. Dicha arteria discurre en dirección ventral en forma de arco emitiendo dos ramas terminales, la A. del conducto deferente (rama craneal) que lo acompaña y nutre, y la A. rectal media (rama caudal), que irriga el recto, próstata y uretra (Evans \& De Lahunta, 2001). Las ramas arteriales entran a la próstata en la superficie dorsal o dorsolateral de la glándula (Smith), siendo la vascularización de los lobos independiente (Stefanov et al., 2000). La A. rectal media, genera tres pequeños vasos hacia la glándula prostática, las Arterias (As.) prostáticas craneal, 
media y caudal (Stefanov). La sangre venosa drena a través de las venas de la próstata y la uretra en la vena ilíaca interna (Freitag et al.; Smith). Los vasos linfáticos prostáticos se vacían hacia los nódulos linfáticos ilíacos (Freitag et al.).

La glándula prostática canina comparte muchas similitudes morfológicas y funcionales con la próstata del humano (Leroy \& Northrup, 2009). Las similitudes de las enfermedades de la próstata entre ambas especies ha llevado a un enorme número de estudios sobre las lesiones de la próstata de los perros (Foster, 2012). El perro, por lo tanto, en general ha sido considerado como un modelo animal adecuado para el estudio de las enfermedades de la próstata en el hombre, como son la hiperplasia benigna y el cáncer, y de esta forma poder desarrollar tratamientos efectivos (Lai et al., 2008; Leroy \& Northrup).

De tal manera que comprender el suministro vascular de la próstata y los tejidos circundantes, es esencial para reducir el riesgo postquirúrgico de incontinencia urinaria, hemorragia grave y necrosis avascular (Freitag et al.). Además, la descripción de la vascularidad normal, debe proporcionar una base morfológica completa y coherente para la detección de neovascularización de la glándula prostática. Siendo ésta un importante indicador diagnóstico y signo de pronóstico de la enfermedad de dicha glándula, como es un tumor prostático (Stefanov).

Por lo cual, el objetivo del presente trabajo es aportar a mejorar el conocimiento sobre la vascularidad arterial extraglandular de la próstata del perro y los vasos que se comparten con otros órganos adyacentes, factor importante a considerar en procedimientos quirúrgicos, tales como, la prostactectomía, resección de quistes y neoplasias.

\section{MATERIAL Y MÉTODO}

Se utilizaron 5 machos caninos mestizos, entre los 3 y 5 años de edad, de un peso entre 15 a $20 \mathrm{~kg}$, sin patologías prostáticas diagnosticadas, obtenidos de clínicas veterinarias particulares y de Departamentos de Sanidad Ambiental, eutanasiados en dichos lugares y trasladados al Laboratorio de Anatomía del Departamento de Patología Animal de la Facultad de Ciencias Veterinarias y Pecuarias de la Universidad de Chile.

Se procedió a la fijación de los animales, con mezcla fijadora-conservadora, mediante perfusión intravascular por gravedad vía A. carótida común. Luego de 72 horas, se procedió a inyectar $150 \mathrm{ml}$ de látex coloreado rojo, a través de la misma vía. Posteriormente, los ejemplares se conservaron a $4^{\circ} \mathrm{C}$, hasta su estudio anatómico.

Se abordó el ejemplar, seccionándolo transversalmente a nivel las últimas vértebras lumbares, se removieron los órganos abdominales hasta despejar vejiga y vaciar colon. Posteriormente, se retiró la sínfisis pélvica y se despejó la próstata, uretra y sus vasos. Se disecó y se observó mediante lupa $5 \mathrm{X}$, los vasos arteriales regionales. Se describieron los vasos visualizados a partir de la A. pudenda interna y las ramas que ingresan a la próstata. Finalmente el material fue conservado por inmersión en formalina al 5\% a temperatura ambiente, hasta la toma de las fotografías.

Los resultados fueron tabulados, comparando los vasos sanguíneos descritos en cada ejemplar, para hacer un enfoque descriptivo y proporcional de acuerdo al número de casos estudiados (Tabla I).

\section{RESULTADOS}

Tabla I. Resumen de arterias comprometidas en la irrigación prostática observadas mediante técnica de inyección intravascular con látex coloreado.

\begin{tabular}{|c|c|c|c|c|c|}
\hline \multirow{2}{*}{$\begin{array}{l}\text { Frecuencia } \\
\text { V asos sanguíneos }\end{array}$} & \multicolumn{5}{|c|}{ Animal No } \\
\hline & 1 & 2 & 3 & 4 & 5 \\
\hline A. pudenda inter na & + & + & + & + & + \\
\hline A. prostática & + & + & + & + & + \\
\hline A. del conducto deferente (rama craneal) & --- & --- & + & + & --- \\
\hline A. vesical caudal & + & + & + & + & --- \\
\hline Rama ureteral & + & --- & + & + & + \\
\hline Rama uretral & --- & --- & + & --- & --- \\
\hline A. rectal media (rama caudal) & --- & --- & + & + & --- \\
\hline A. prostática craneal & + & + & + & + & + \\
\hline A. prostática media & + & + & + & + & + \\
\hline A. prostática caudal & + & + & + & + & + \\
\hline
\end{tabular}

Se observó que en 2 de los 5 animales, la A. prostática discurrió en dirección ventral originando la A. del conducto deferente (rama craneal) y la A. rectal media (rama caudal) (Figs. 1 y 2) y en 3 de 5 animales, la A. prostática generó una sola rama (Fig. 3).

En 2 de 5 perros la A. prostática craneal, provino de la A. del conducto deferente y las As. 
prostáticas media y caudal, de la A. rectal media (Figs. 1 y 2). En los otros 3 ejemplares, las As. prostáticas craneal, media y caudal, se originaron directamente de la A. prostática no evidenciándose las As. del conducto deferente y rectal media (Fig. 3).

En 4 de 5 animales, la A. del conducto deferente, derivó hacia vejiga (Figs. 1 y 2) y en 2 de ellos, derivó a uréteres (Fig. 1). Finalmente, en 1 perro, la A. rectal media derivó hacia la uretra (Fig. 1).

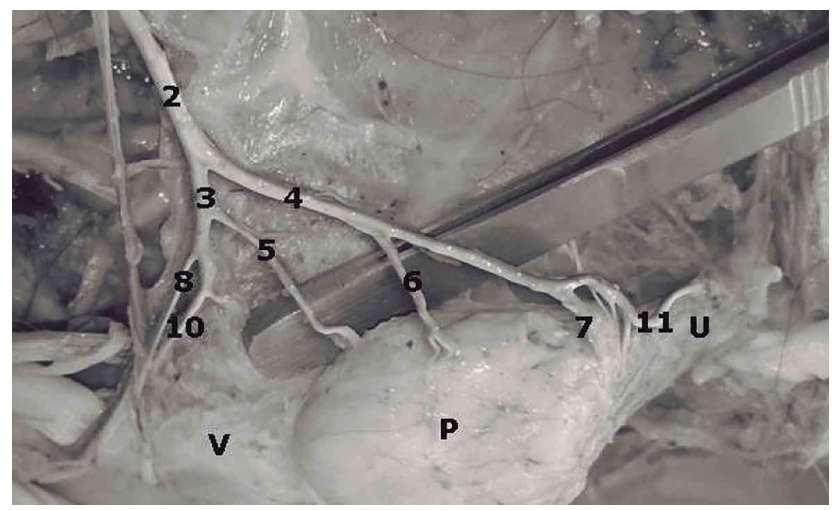

Fig. 1. Arterias que participan en la irrigación prostática observadas mediante técnica de inyección intravascular con látex coloreado (2X). 2) A. Prostática, 3) A. del Conducto Deferente (Rama craneal), 4) A. Rectal Media (Rama caudal) 5) A. Prostática craneal, 6) A. Prostática media, 7) A. Prostática caudal, 8) A. Vesical caudal, 10) Rama ureteral 11) A. Uretral, P) Próstata, U) Uretra, V) Vejiga

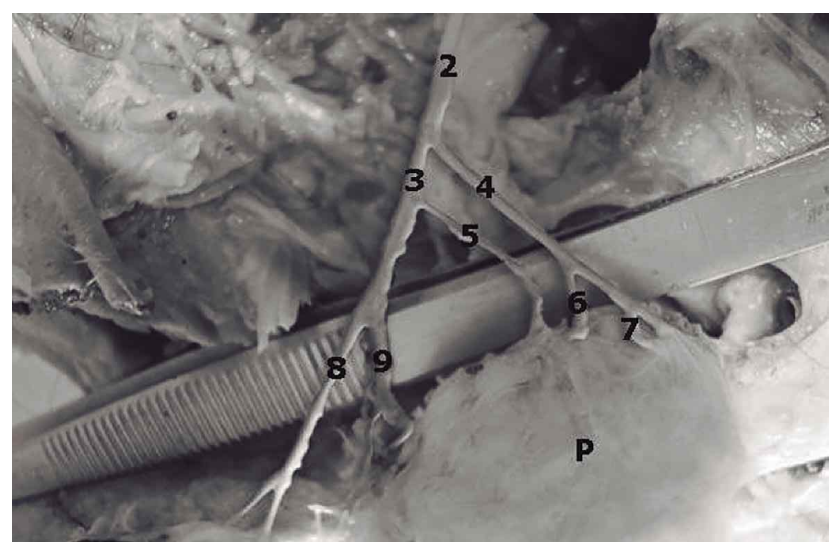

Fig. 2. Arterias que participan en la irrigación prostática observadas mediante técnica de inyección intravascular con látex coloreado (2X). 2) A. Prostática, 3) A. del Conducto Deferente (Rama craneal), 4) A. Rectal Media (Rama caudal) 5) A. Prostática craneal, 6) A. Prostática media, 7) A. Prostática caudal, 8) A. Vesical caudal, 9) Rama uretral, P) Próstata.

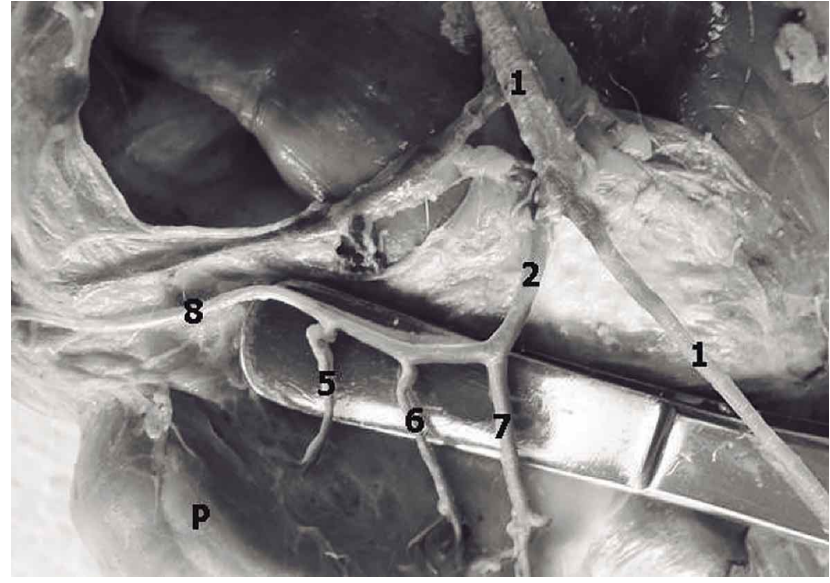

Fig. 3. Arterias que participan en la irrigación prostática observadas mediante técnica de inyección intravascular con látex coloreado (2X). 1) A. Pudenda Interna, 2) A. Prostática, 5) A. Prostática craneal, 6) A. Prostática media, 7) A. Prostática caudal, 8) A. Vesical caudal, P) Próstata.

\section{DISCUSIÓN}

Describir la distribución de los vasos sanguíneos de la próstata canina es importante y útil para la comprensión del control fisiológico y ciertos procesos patológicos de dicha glándula. La glándula prostática canina, comparte muchas similitudes morfológicas y funcionales con la próstata humana. Por lo cual, los perros afectados con hiperplasia benigna o cáncer de próstata, son modelos relevantes para esta enfermedad en el hombre (Leroy \& Northrup).

Tomando como referencia lo indicado por Evans \& De Lahunta, en el sentido que el aporte arterial de la próstata deriva de la A. ilíaca interna, que se continúa en la A. pudenda interna, la cual es un vaso más pequeño y ventral que corre en dirección caudal sobre el tendón terminal del M. psoas menor y que a nivel de la Art. sacro ilíaca, formando un ángulo de $45^{\circ}$, da origen a la A. prostática, la que discurre en dirección ventral en forma de arco emitiendo dos ramas terminales, una craneal, la A. del conducto deferente y una caudal, la A. rectal media, que irriga el recto, próstata y uretra. Posteriormente, de la A. rectal media se originan las As. prostáticas craneal, media y caudal, que ingresan a la cápsula de la glándula. Lo que corrobora lo descrito por Stefanov et al. (2000).

En el presente trabajo se observó que independiente de las ramificaciones, la A. prostática ingresa siempre a la cápsula de la glándula mediante las As. prostáticas craneal, media y caudal, lo que corrobora lo descrito por Stefanov. 
A diferencia del perro, la A. prostática del hombre, proviene de distintas arterias, siendo el origen más frecuente la A. pudenda interna y el Tronco gluteal pudendo y menos frecuentes de las As. obturatriz o A. gluteal inferior (Bilhim et al., 2011).

Por otro lado, en el conejo la A. prostática, surge de la bifurcación entre la A. ilíaca externa e interna (Kürtül et al., 2001). En el cerdo, la A. prostática proviene de la A. vesical inferior (Sun et al., 2008). En la rata, el suministro de sangre a la próstata proviene de la A. vesical que se origina de la A. ilíaca interna (McVary et al., 1994), Finalmente en el gato la A. prostática se deriva de la A. pudenda interna, y desde ésta surgen las As. vesicales craneal y caudal que irrigan al conducto deferente, la uretra y la próstata (Erdogan, 2011).

Los resultados de este estudio describen que la A. pudenda interna da origen a la A. prostática. La A. prostática, en analogía con lo indicado en NAV, corre en dirección ventral originando la A. del conducto deferente (rama craneal) y la A. rectal media (rama caudal). Debido a que la A. prostática se divide en dos grandes ramas, una hacia la vejiga y otra hacia la próstata; Stefanov et al. (2000), propuso que la arteria que se origina en la A. pudenda interna, se debería llamar "Arteria Pelviana". La A. rectal media irriga uretra, recto y próstata, esta última, antes de ingresar a la glándula, se divide en 3 vasos llamados: As. prostáticas craneal, media y caudal (Stefanov). Esto difiere con lo que indica la NAV, asunto que está en discusión actualmente. De acuerdo con el presente estudio, los resultados encontrados corresponden a los sugeridos por Stefanov et al. (1999).

\section{AGRADECIMIENTOS}

Sr. Luis Muñoz Díaz, por la colaboración en la preparación del material anatómico.

OLIVARES, R. \& AGUAYO, M. Extra glandular arterial vascularity of the dog prostate. Int. J. Morphol., 32(4):1277-1281, 2014.

SUMMARY: The prostate is the dog's most important accessory sex gland, located in the pelvic canal, retroperitoneal and flow to the bladder, completely wrapping the pelvic urethra on its way out of the bladder neck. Its function is to produce seminal fluid that provides an optimum environment for the survival and motility. It may be affected by a number of pathological processes such as benign prostatic hyperplasia and neoplasia. The aim of this study was to describe the distribution of extraglandular arterial blood vessels of the canine prostate, as a factor of importance and relevance to the understanding of physiological control and certain pathological processes of the gland for subsequent medical and surgical approach. Five adult no breed male dogs between 3 and 5 years old, weighing 15 to 20 $\mathrm{kg}$ with no diagnosed prostatic disease, were used. They were euthanized in private and public veterinary clinics. The animals were perfused through the common carotid artery with fixative-preservative solution, and were injected by the same route after 72 hours, with red colored latex keeping the bodies in refrigeration until regional dissection. In relation with some authors, it was determined that independent of the ramifications of the prostatic artery, it always irrigates the gland through three vascular branches.

KEY WORDS: Prostate; Irrigation; Dog.

\section{REFERENCIAS BIBLIOGRÁFICAS}

Bilhim, T.; Pisco, J. M.; Furtado, A.; Casal, D.; Pais, D.; Pinheiro, L. C. \& O'Neill, J. E. Prostatic arterial supply: demonstration by multirow detector angio CT and catheter angiography. Eur. Radiol., 21(5):1119-26, 2011.

Dimitrov, R.; Yonkova, P.; Vladova, D. \& Kostov, D. Computed tomography imaging of the topographical anatomy of canine prostate. Trakia J. Sci., 8(2):78-82, 2010.

Erdogan, S. Distribution of the arterial supply to the lower urinary tract in the domestic tom-cat (Felis catus). Vet. Med-Czech., 56:202-8, 2011.
Evans, H. \& De Lahunta, A. Guide to the dissection of the dog. 5a ed. México D.F., McGraw-Hill, 2002.

Freitag, T.; Jerram, R. M.; Walker, A. M. \& Warman, C. G. Surgical management of common canine prostatic conditions. Compend. Contin. Educ. Vet., 29(11):656-8, 2007.

Foster, R. A. Common lesions in the male reproductive tract of cats and dogs. Vet. Clin. North Am. Small Anim. Pract., 42(3):527-45, 2012.

Gobello, C. \& Corrada, Y. Noninfectious prostatic disease in dogs. Compend. Contin. Educ. Vet., 24:99-107, 2002. 
International Committee on Veterinary Gross Anatomical Nomenclature (I.C.V.G.A.N.). Nómina Anatómica Veterinaria. 5th ed. Knoxville, Editorial Committee Hannover, Columbia, Gent, Sapporo, 2005.

Kürtül, I' ; Özgel, Ö.; Dursun, N. \& Bozkurt, E. Ü. Morphology of the accessory sex glands and their vascularization in New Zealand rabbits. Kafkas Üniv. Vet. Fak. Derg., 7:95-9, 2001.

Kustritz, R. Medical treatment of canine prostate disease. St. Paul MN, University of Minnesota College of Veterinary Medicine 50th Anniversary Symposium, 1997.

Lai, C. L.; van den Ham, R.; van Leenders, G.; van der Lugt, J. \& Teske, E. Comparative characterization of the canine normal prostate in intact and castrated animals. Prostate, 68(5):498507,2008 .

Leroy, B. E. \& Northrup, N. Prostate cancer in dogs: comparative and clinical aspects. Vet. J., 180(2):149-62, 2009.

McVary, K. T.; Razzaq, A.; Lee, C.; Venegas, M. F.; Rademaker, A. \& McKenna, K. E. Growth of the rat prostate gland is facilitated by the autonomic nervous system. Biol. Reprod., 51(1):99-107, 1994

Smith, J. Canine prostatic disease: a review of anatomy, pathology, diagnosis, and treatment. Theriogenology, 70(3):375-83, 2008.

Stefanov, M. Extraglandular and intraglandular vascularization of canine prostate. Microsc. Res. Tech., 63(4):188-97, 2004.

Stefanov, M.; Martín-Alguacil, N. \& Martín-Orti, R. Distinct vascular zones in the canine prostate. Microsc. Res. Tech., 50(2):169-75, 2000.

Stefanov, M.; Martín-Orti, R.; Martín, R. \& Gaspar, I. Main blood vessels of the canine prostatic gland. Bulg. J. Agric. Sci., 5:78995, 1999.

Sun, F.; Sánchez, F. M.; Crisóstomo, V.; Lima, J. R.; Luis, L.; García-Martínez, V.; López-Sánchez, C.; Usón, J. \& Maynar, M. Benign prostatic hyperplasia: transcatheter arterial embolization as potential treatment--preliminary study in pigs. Radiology, 246(3):783-9, 2008.

Thibaut, J.; Santander, J. \& Mieres, M. Estudio comparativo de la próstata en perros mediante ecografía transrectal y transabdominal. Arch. Med. Vet., 41(1):61-6, 2009.

\author{
Dirección para Correspondencia: \\ Dr. Ricardo Olivares Pérez-Montt \\ Departamento de Patología Animal \\ Facultad Ciencias Veterinarias y Pecuarias \\ Universidad de Chile \\ Santiago \\ CHILE
}

Email: rolivare@uchile.cl

Recibido: 07-08-2014

Aceptado: 27-09-2014 